Journal of Business \& Management (COES\&RJ-JBM)

ISSN (E): 2306-7179 ISSN (P): 2306-8043

Publisher: Centre of Excellence for Scientific \& Research Journalism, COES\&RJ LLC

Online Publication Date \& Issue: 1st April 2019, Vol.7, No.2, April 2019

https://doi.org/10.25255/jbm.2019.7.2.95.110

\title{
Job Commitment and its Relation to Organizational Justice: A Theoretical
} Perspective

Yazan Taher Shawabkeh

An Independent Researcher, Amman, Jordan, yazanshwabkeh@gmail.com

Musa Al-Lozi

Department of Public Administration, School of Business, The University of Jordan, Amman, Jordan \& President of Aqaba Branch, The University of Jordan, Aqaba,

Lozi.musa@ju.edu.jo

\begin{abstract}
:
The organizations are based on a number of foundations, one of which is financial and non-financial resources. Non-financial resources include human resources and nonhuman resources. The human resource is distinguished from all other resources of the organization by reason and by its psychological and social dimensions. Organizations therefore need personnel who are psychologically linked to their work in the present and future. And that no governmental or private organization can develop its efficiency and effectiveness without the will of its staff to cooperate with it and their desire to do so, unless, and to the extent that it has effective and competent staff. The present study aims at reviewing the Arabic and foreign studies related to the job commitment and its relation to organizational justice.
\end{abstract}

\section{Keywords:}

Job Commitment, Organizational Justice, Theoretical Perspective

\section{Citation}

Shawabkeh, Yazan Taher; Al-Lozi, Musa (2019); Job Commitment and its Relation to Organizational Justice: A Theoretical Perspective ; Journal of Business \& Management (COES\&RJ-JBM); Vol.7, No.2, pp.95-110, https://doi.org/10.25255/jbm.2019.7.2.95.110.

This work is licensed under a Creative Commons Attribution 4.0 International License 


\author{
الالتزام الوظيفي وعلاقته بالعدالة التنظيمية: إطار نظري \\ يزن طاهر الثوابكة¹ ، أ.د. موسى اللوزي 2
}

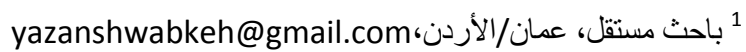

Lozi.musa@ju.edu.jo نائب رئيس الجامعة الاردنية لثؤون المر اكز وخدمة المجتمع/ رئيس فرع العقبة،

\begin{abstract}
الملخص
\end{abstract}

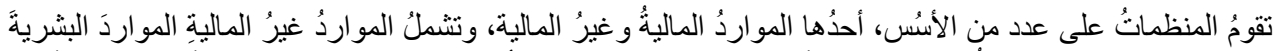

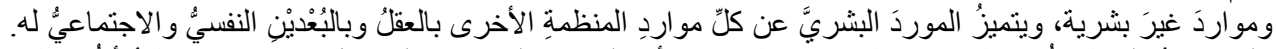

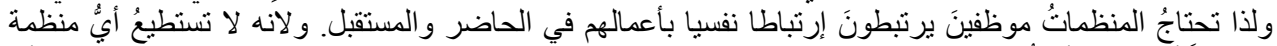

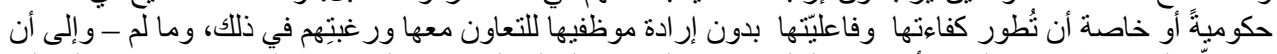

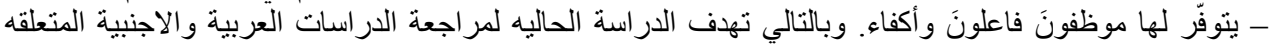

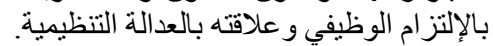
كلمات مفتاحية: الإلتز ام الوظيكي، بالعية العدالة التنظيمية، إطار نظري.

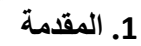

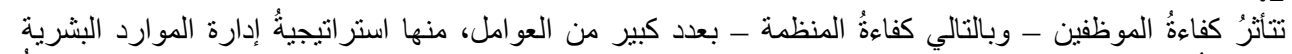

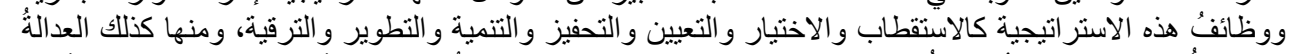

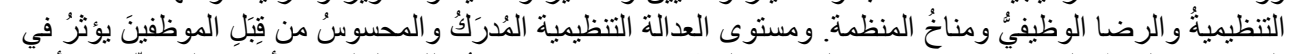

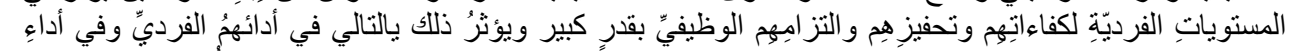

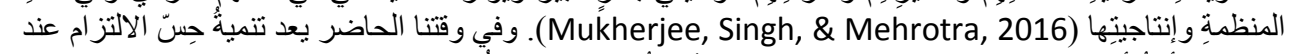

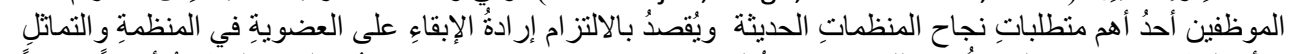

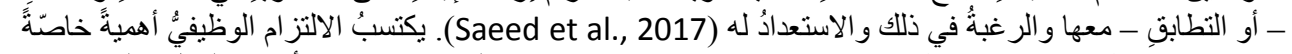

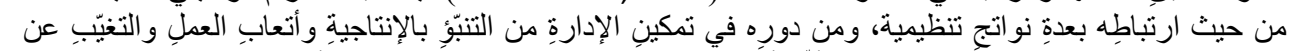

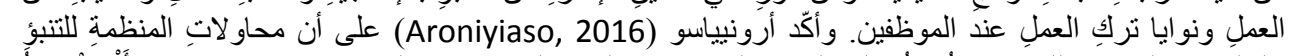

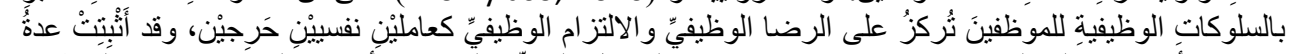

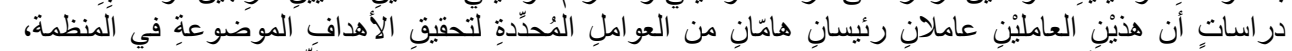

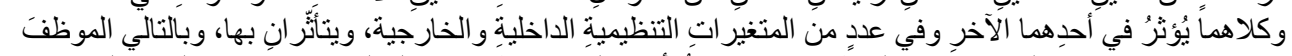

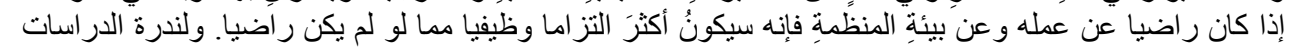

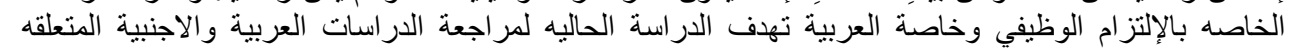
2. بالإلتزام الوظيفي.

2.1 يناقش الباحثون في الأبواب التالية العدالة التنظيمية؛ تعريفها ومداخلها و أهميتها، و العو امل المؤثرة فيها وتللك المتأثرة

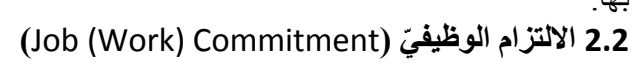

2.2.1 تعريف الالتزام الوظيفيّ (الوني

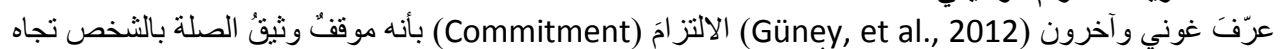

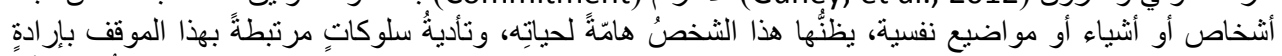

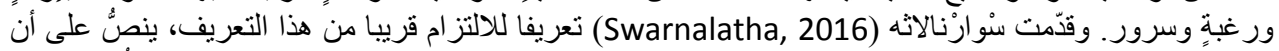

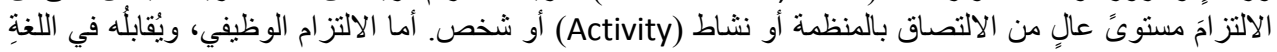

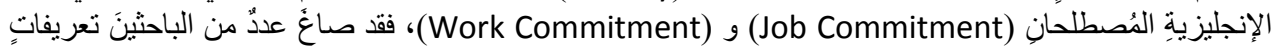

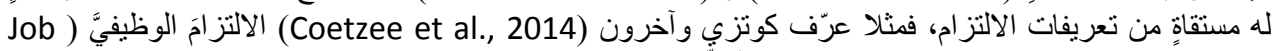
(Commitment

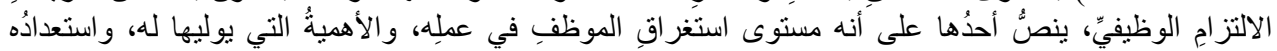

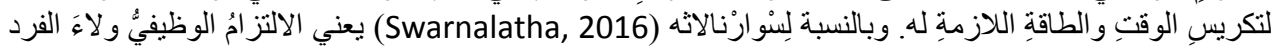

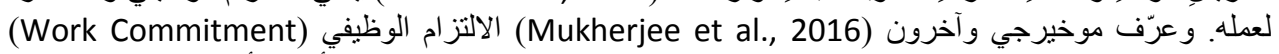
بالإحساس بالمسؤولية عند الفرد تجاه عمله وتجاه المهام التتظيمية الموكلة إليه، و وعرّفوه أيضا بأنه مدى إر ادة الموظف 


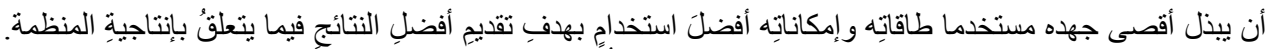

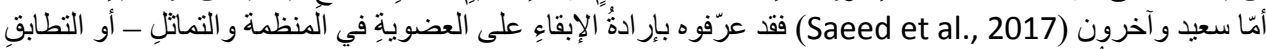
- معها و الر غبةُُ في ذلك و والاستعدادُ له.

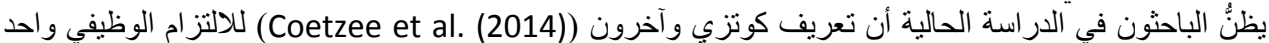

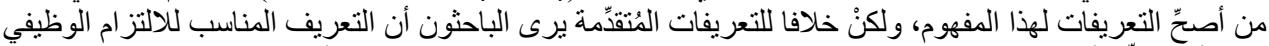

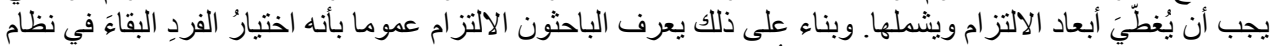

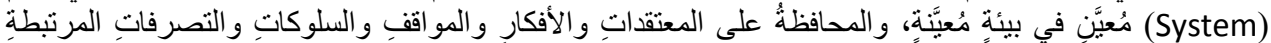

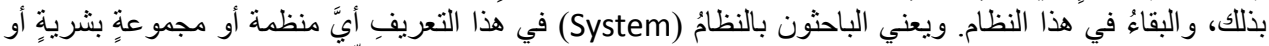

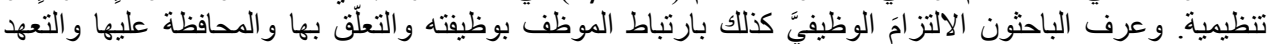

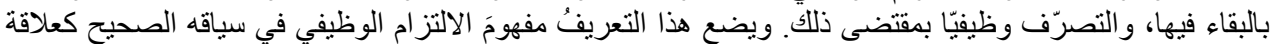

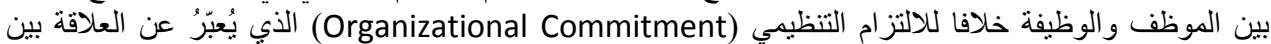

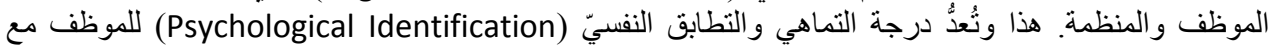

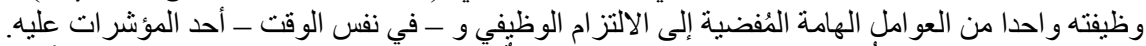

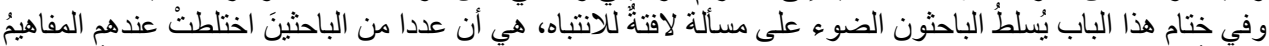

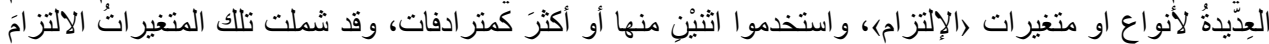

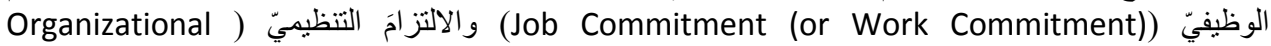

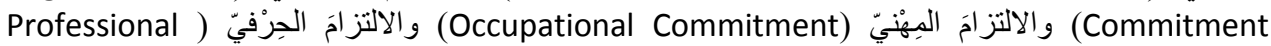

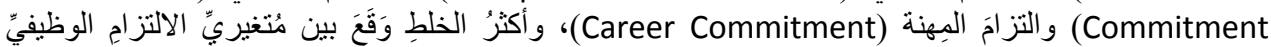

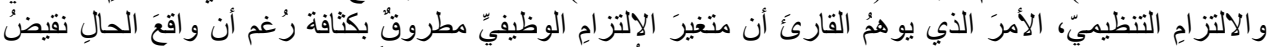

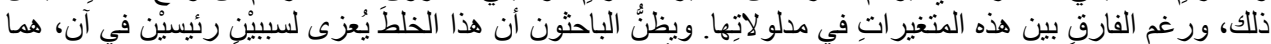

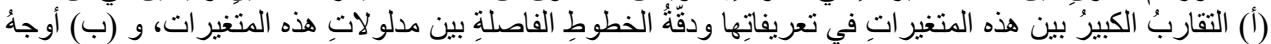

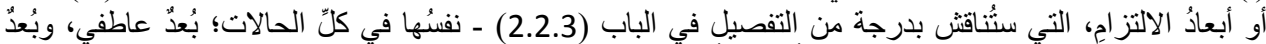

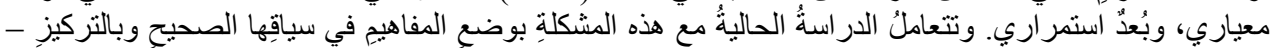

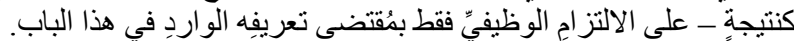

2.2.2

أشتار أَوان (Awan, 2018) إلى أن التوصيف النظري لمحدِّات الالتزام الوظيفي يقوم على منهجيْن، هما المنهج

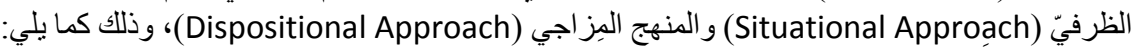

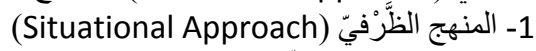

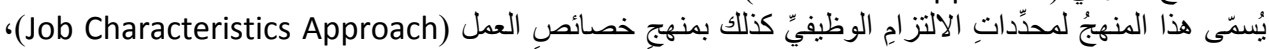

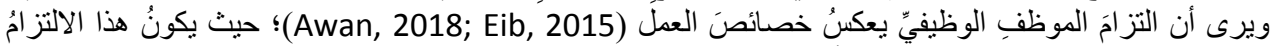

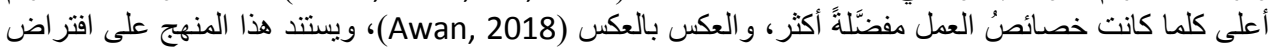

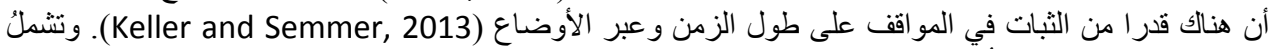

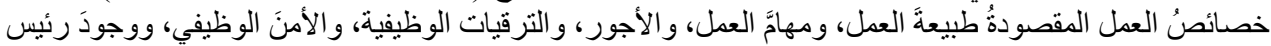

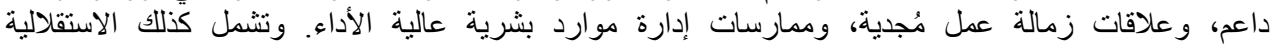
(Autonomy) ) أو السيطرة على العمل (Keller and Semmer, 2013).

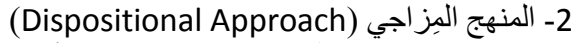

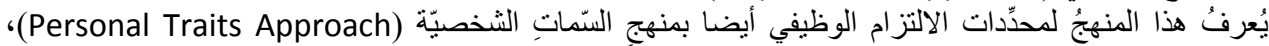

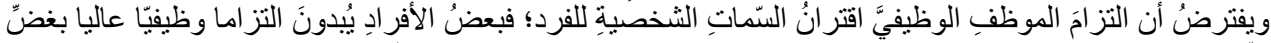

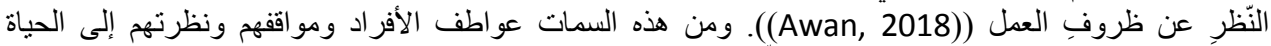

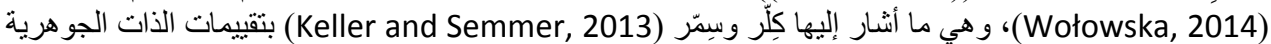

.(Core Self Evaluations)

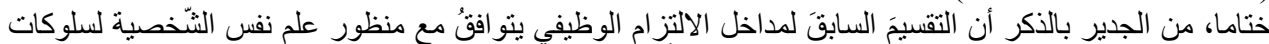

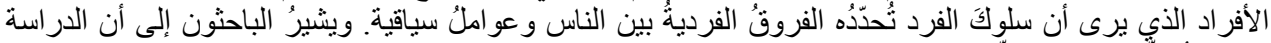

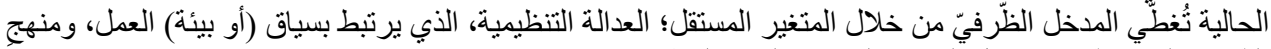

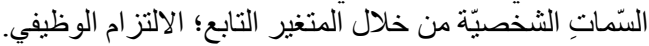

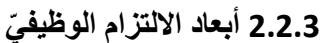

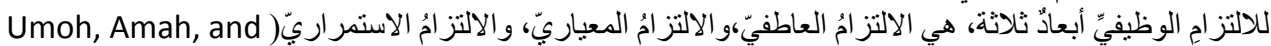

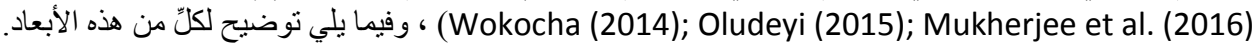


1 الالنز امُ العاطفيُ (Affective Commitment)

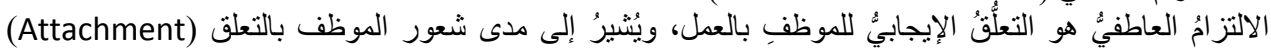

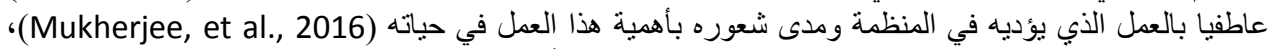

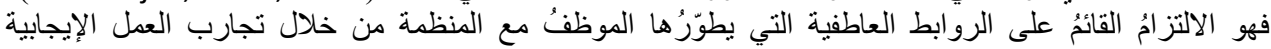

.(Oludeyi, et al., 2018)

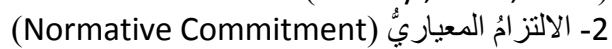

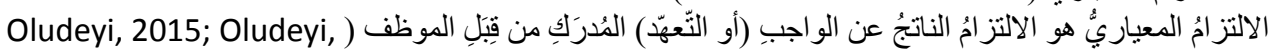

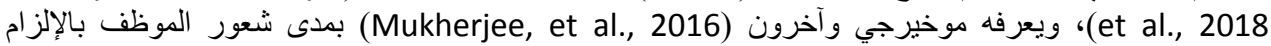

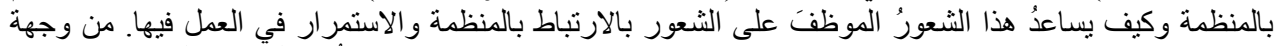

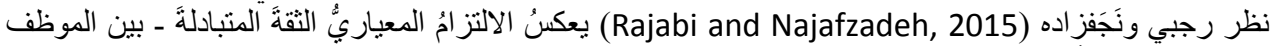

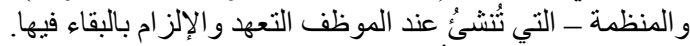

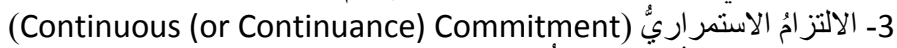

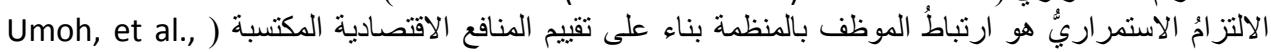

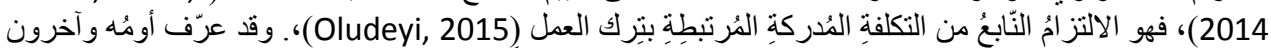

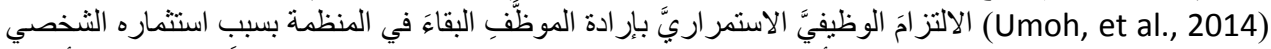

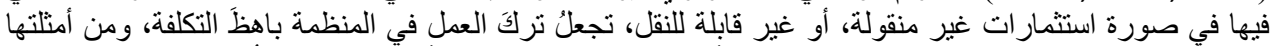

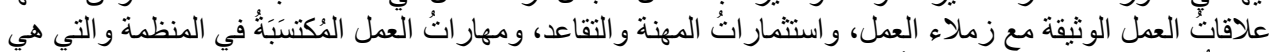

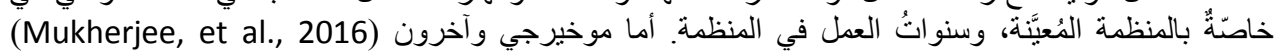

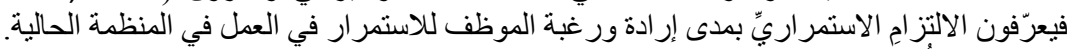

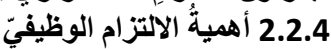

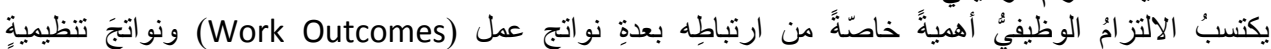

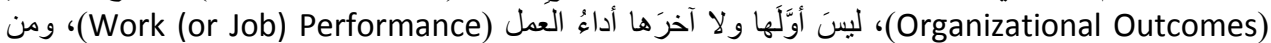

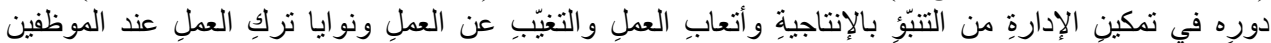
Aroniyiaso, (Agarwal, 2015; Aroniyiaso, 2016; Olaojo and Oyeboade, 2016)

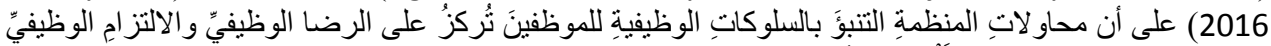

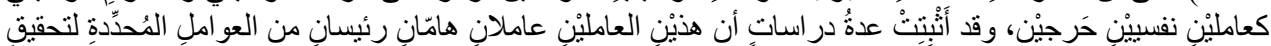

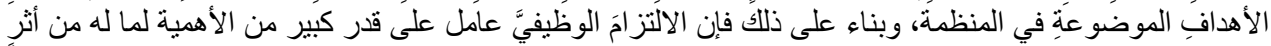

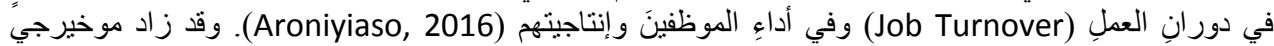

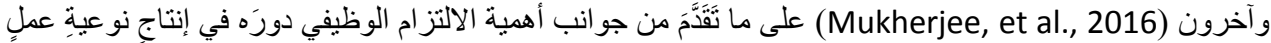

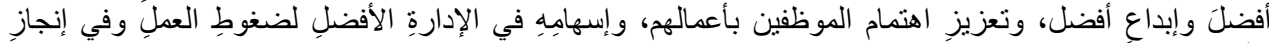

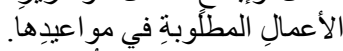

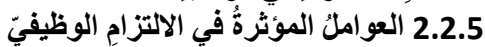

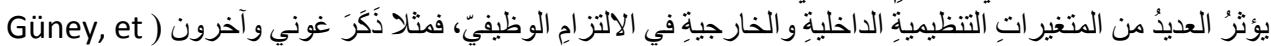

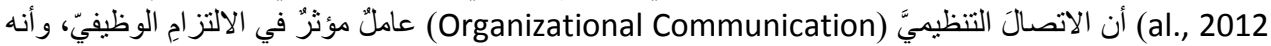

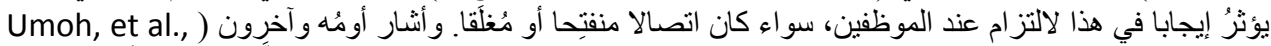

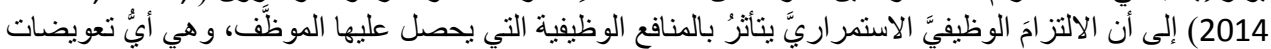

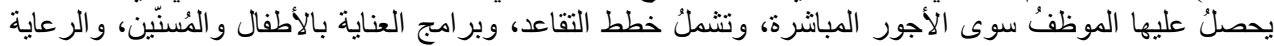

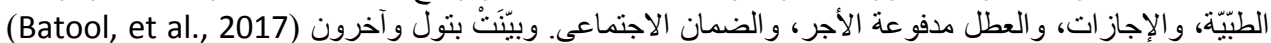

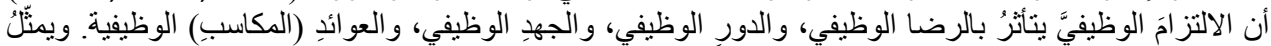

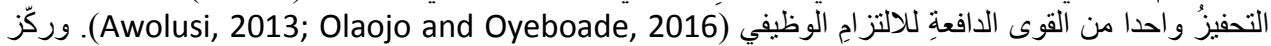

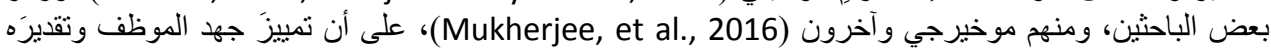
(Recognition)

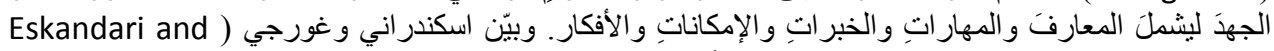

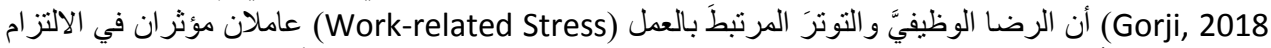

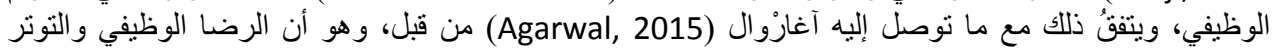
(Stress)

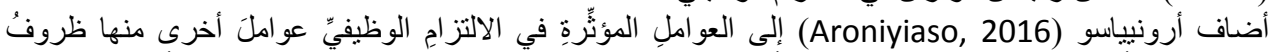

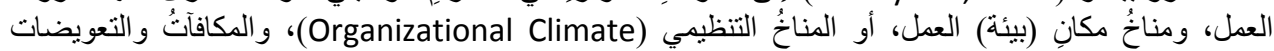




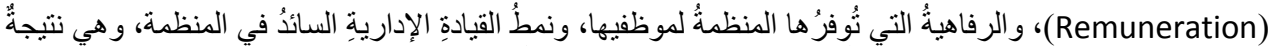

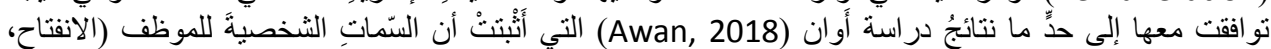

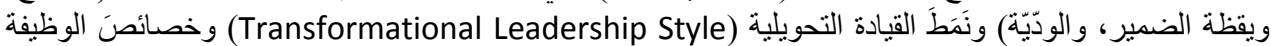

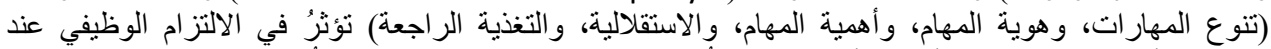

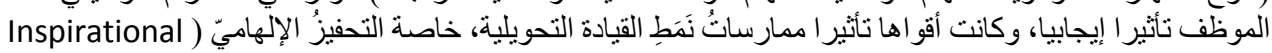
Motivation

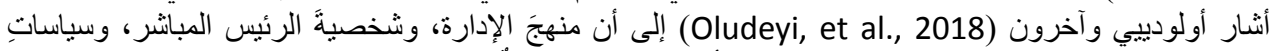

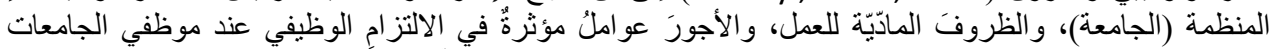

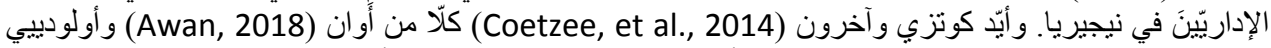

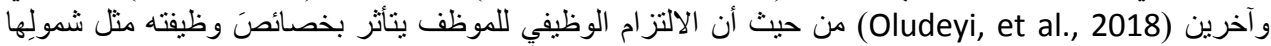

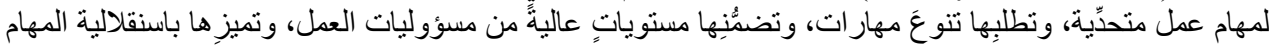

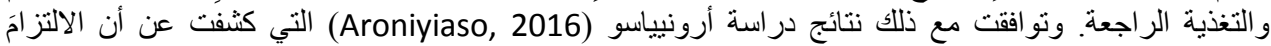

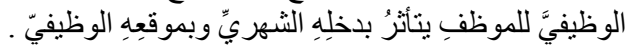

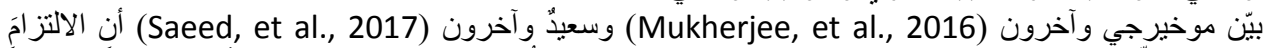

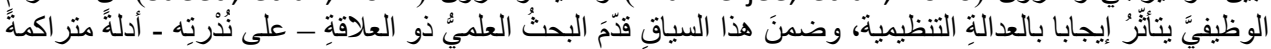

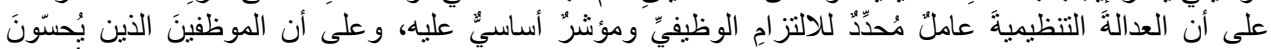

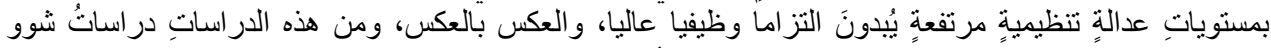

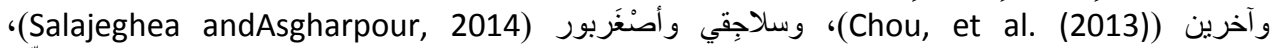

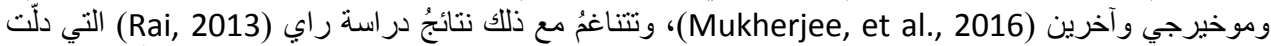

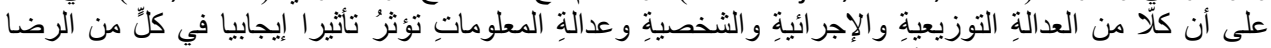

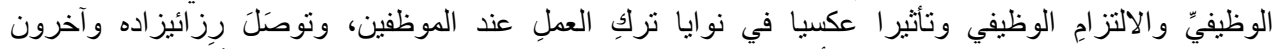
(Rezaeizadeh, et al., 2015)

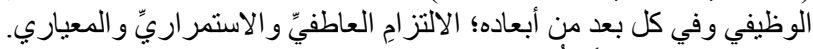

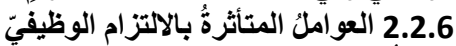

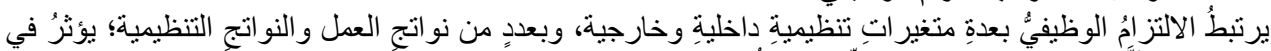

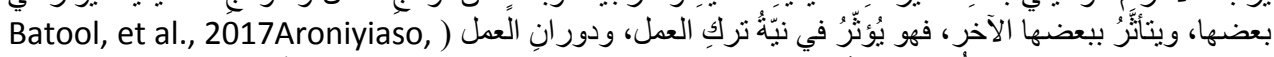

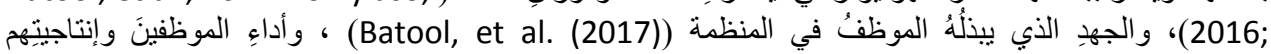
Awolusi, 2013; Agarwal, 2015; Aroniyiaso, 2016; Swarnalatha, 2016; Batool, et al., ) 2017). وأوضح كونزي وآخرون (Coetzee, et al., 2014) أن الالتزامَ الوظيفيّ يؤثرُ في ارتباط الموظف بعمله

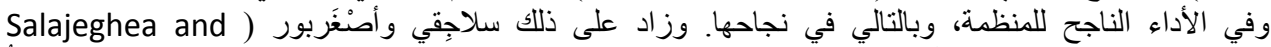

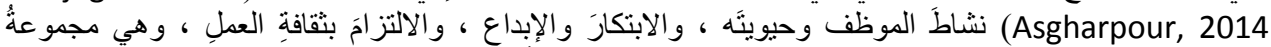

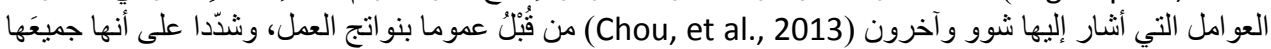

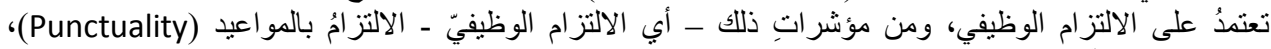

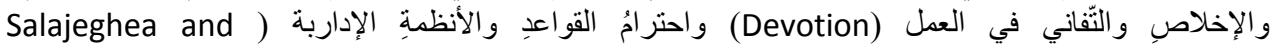

(Asgharpour, 2014; Ajadi and Alade-Yussuf, 2016

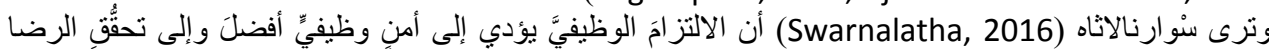

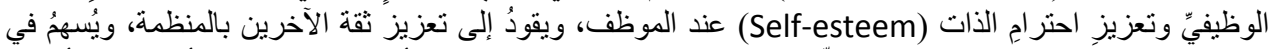

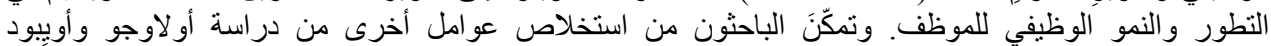

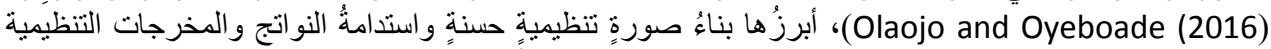

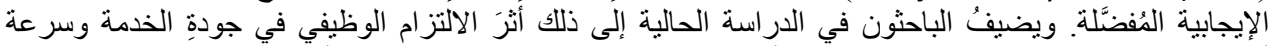

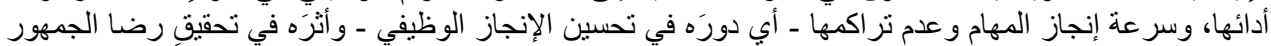

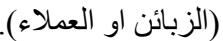

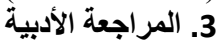

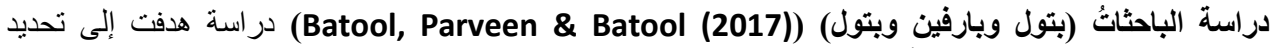

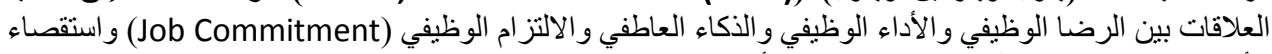

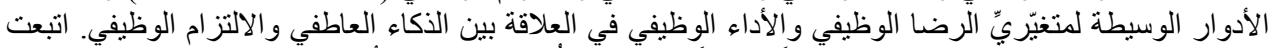

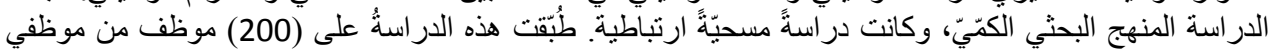




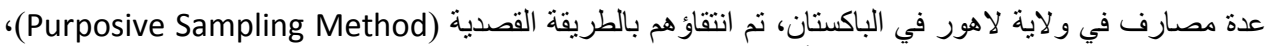

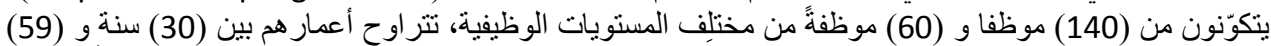

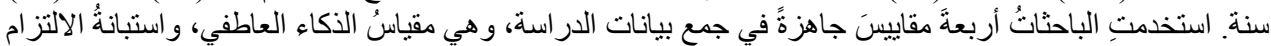

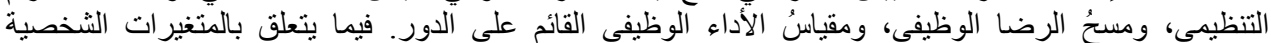
للمبحوثين، والتي كانت الجنسَ والحالةً الزواجيةً (Demographic Variables)

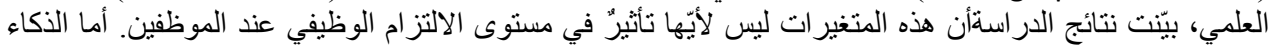

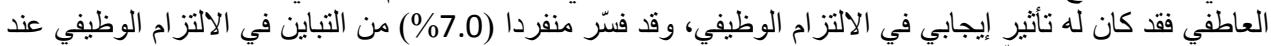

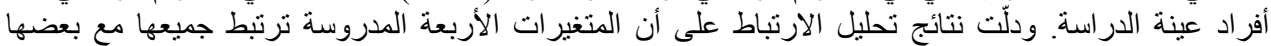

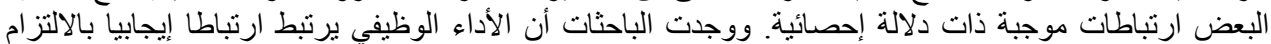

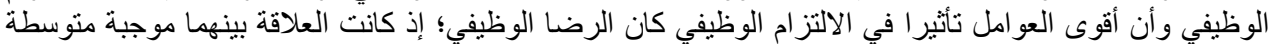

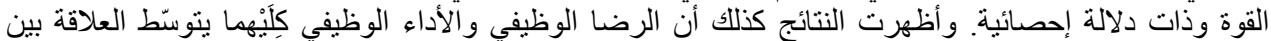

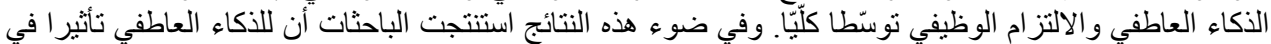
الالتز ام الوظيفي ولكنه تأثير" غير" مبانشر.

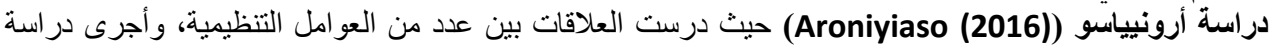

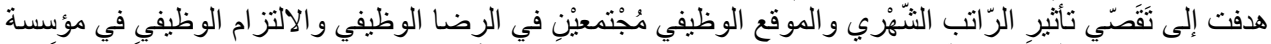

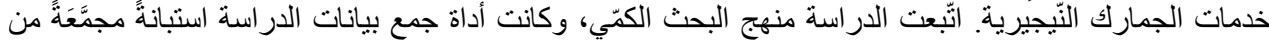

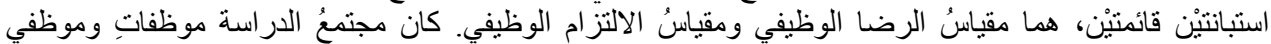

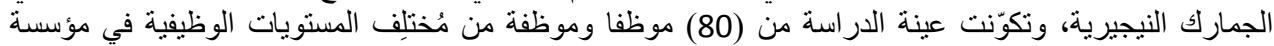

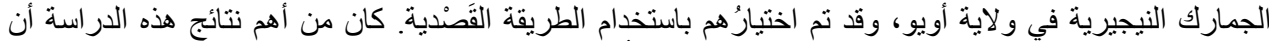

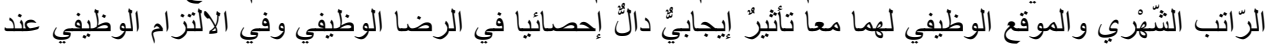

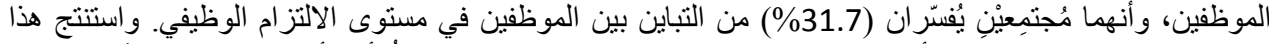

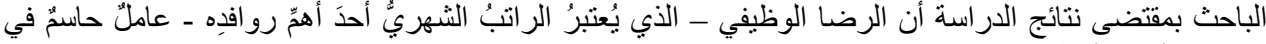

تحقيق الالتز ام الوظيفي.

دراسة كوتزي وسرويدر وتلادنيان (Coetzee, Schreuder, \& Tladinyane (2014) كان هدفها تقديرَ الدور

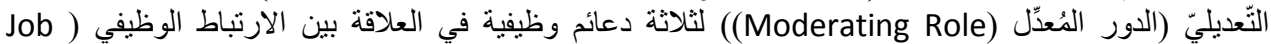

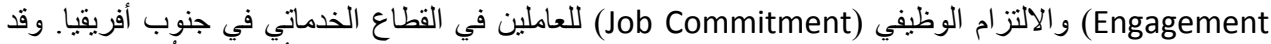

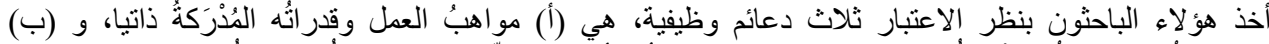

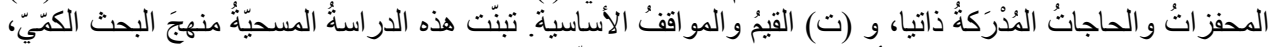

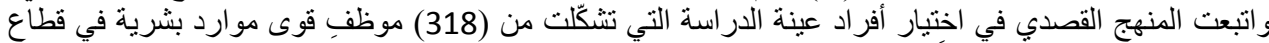

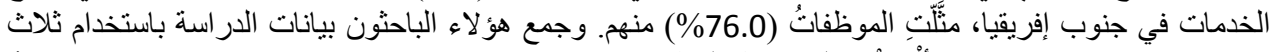

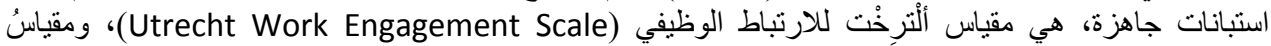

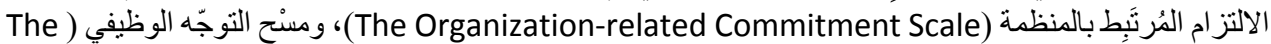
Career Orientations Inventory

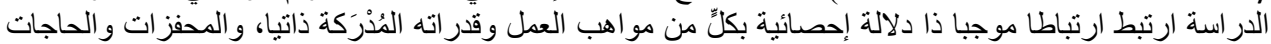

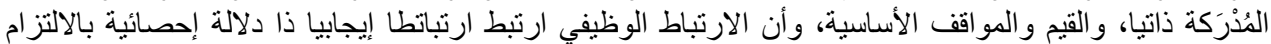

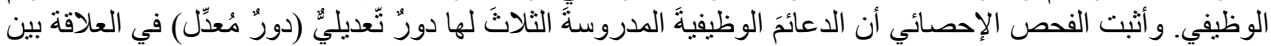

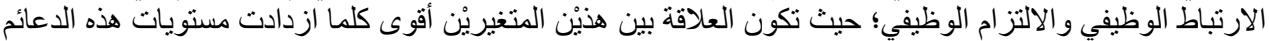

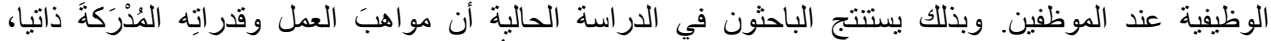

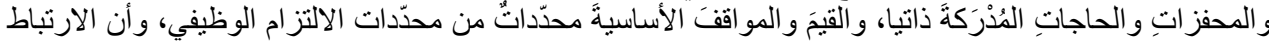
الوظيفي تابعٌ للالتز ام الوظيفي. دراسة موخيرجي وآخرون (Mukherjee et al. (2016) في العلاقات بين العدالة التنظيمية والالتزام الوظيفي

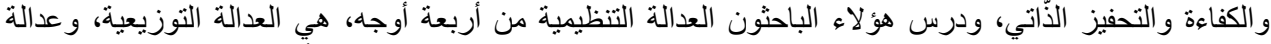

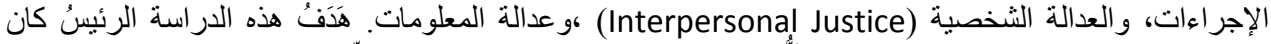

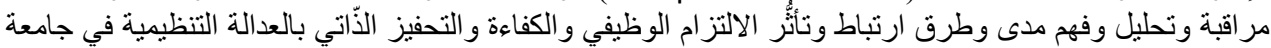

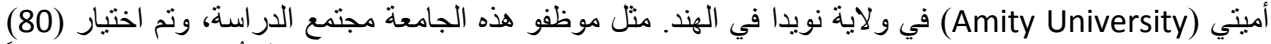

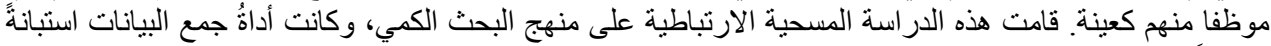

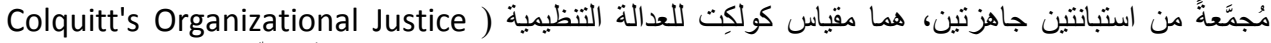

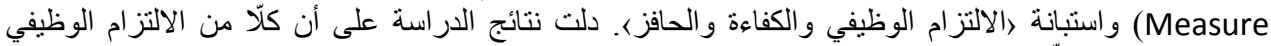
و الكفاءة و التحفيز الذّاتي يرتبط بالعدالة التنظيمية ارتباطا موجبا ذا دلالة إحصائية. 
دراسة (سلاجِقي وأصنغَربور) (Salajeghea \& Asgharpour (2014) تمت هذه الدراسة بهدف فحص العلاقة

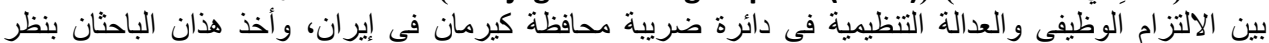

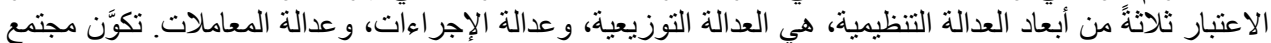

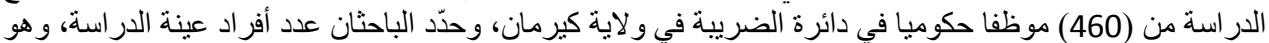

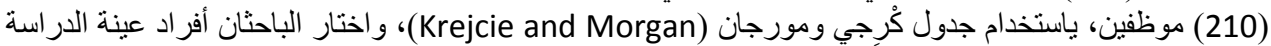

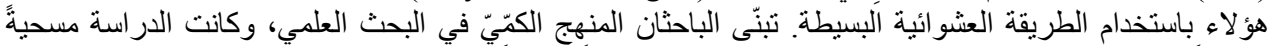

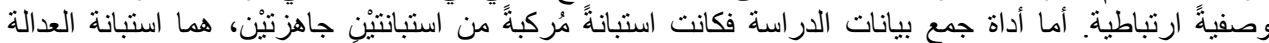

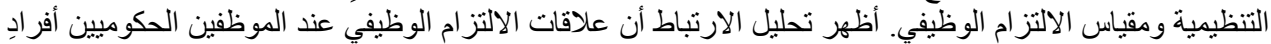

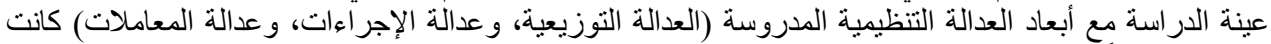

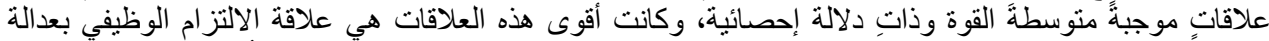

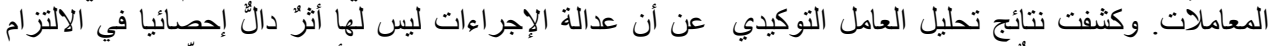

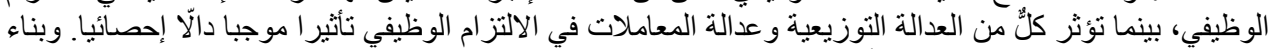

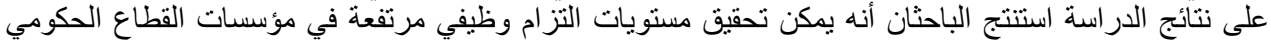

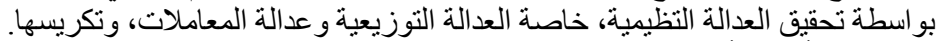

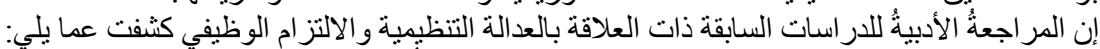

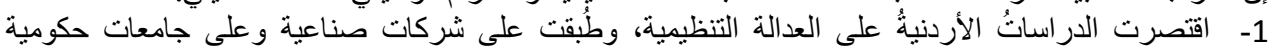

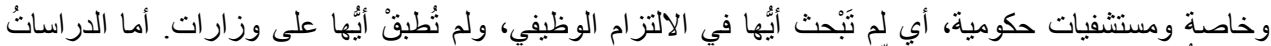

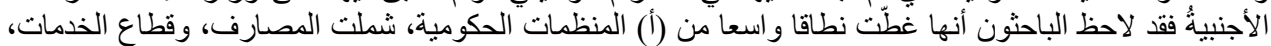

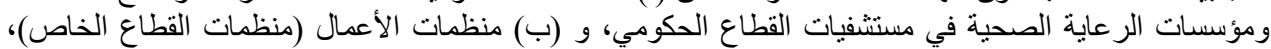
ومنها المصارف، وقطاع الرعاع الخدمات، وجات وجامعات خاصنة.

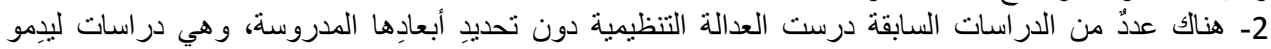

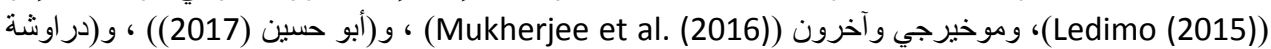

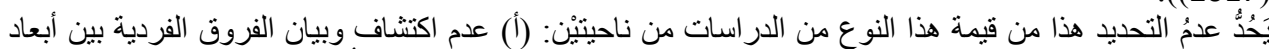

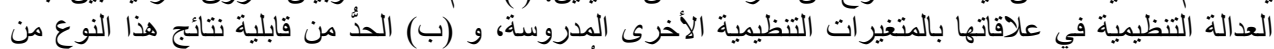

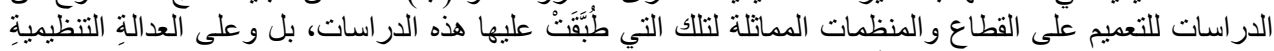

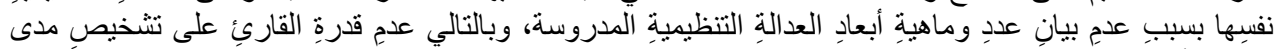
تمثيلِ الأبعادِ المدروسةِ للعدالة التنظيمية.

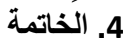

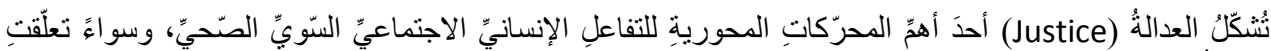

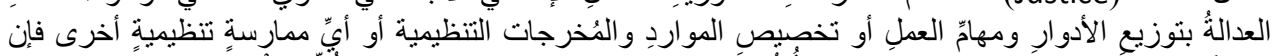

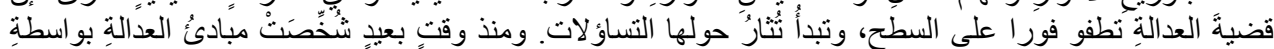

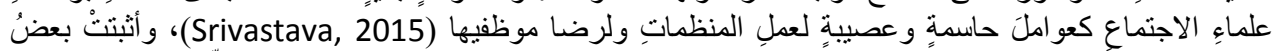

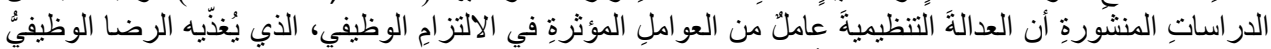

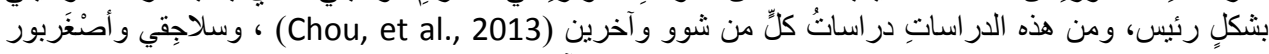

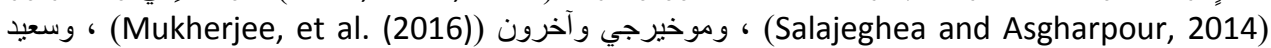

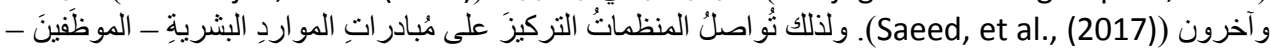

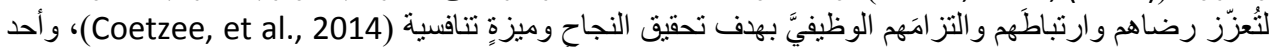

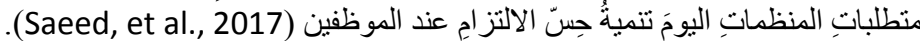

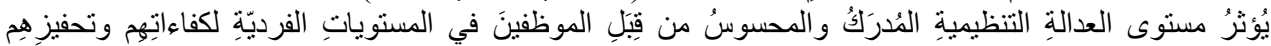

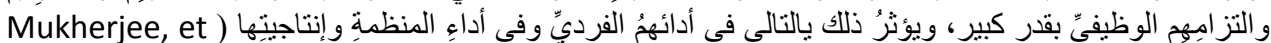

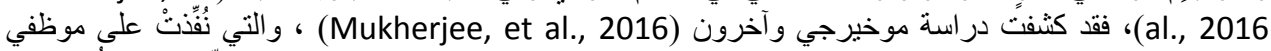

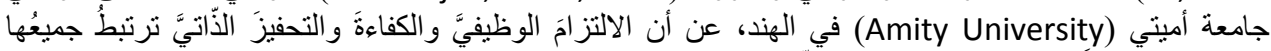

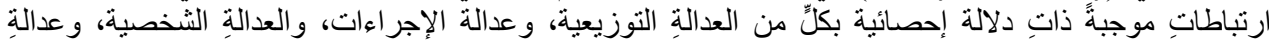

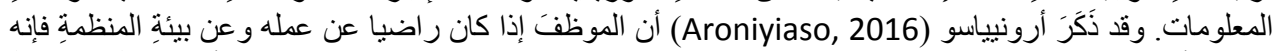

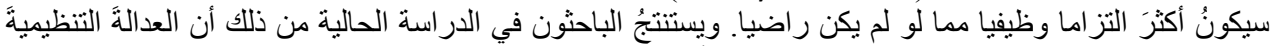

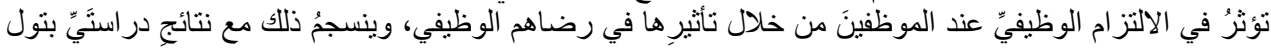




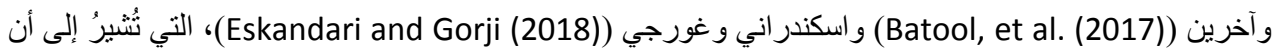

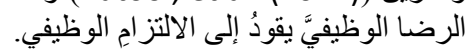

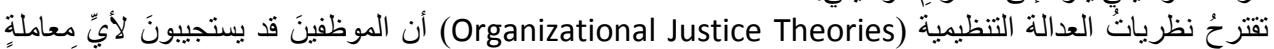

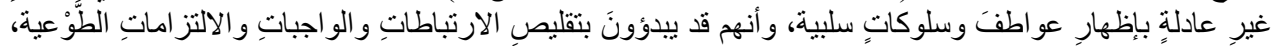

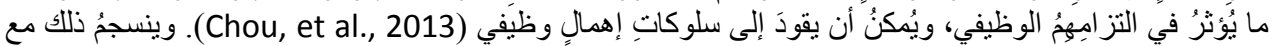

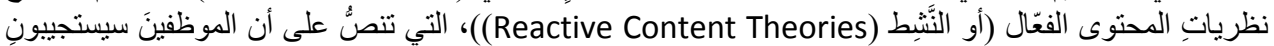

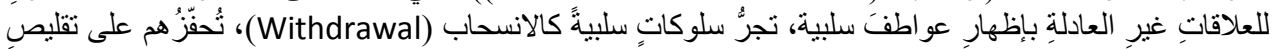

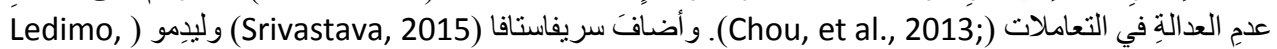

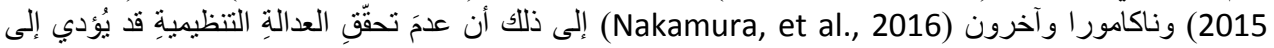

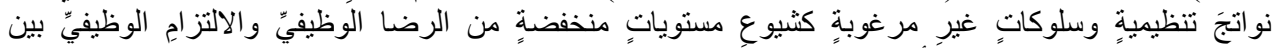

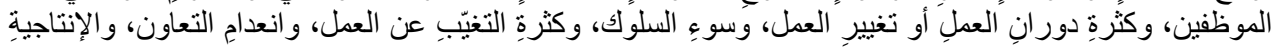

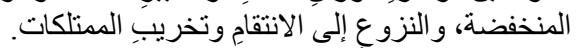

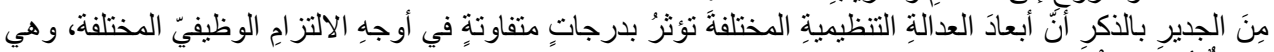

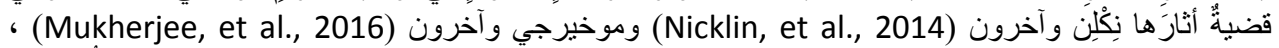

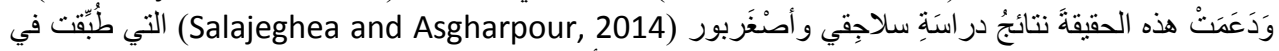

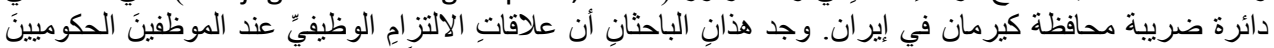

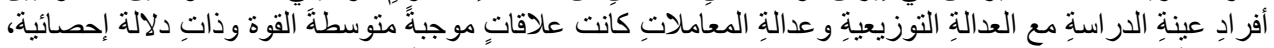

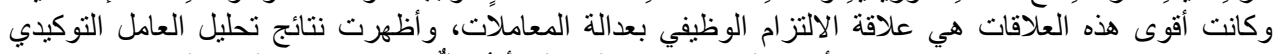

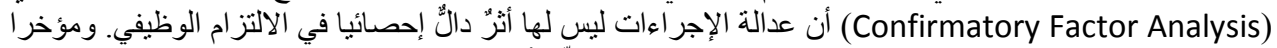

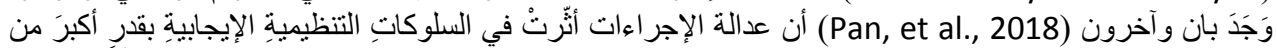

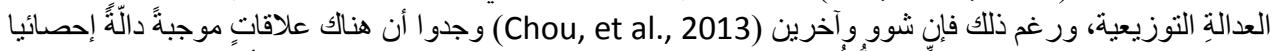

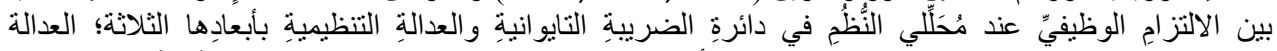

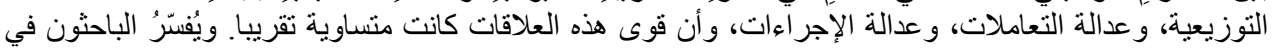

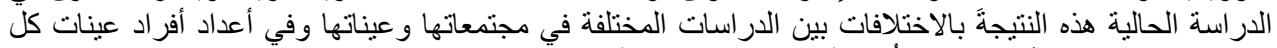

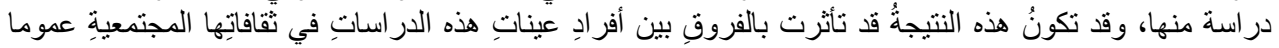

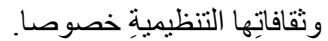

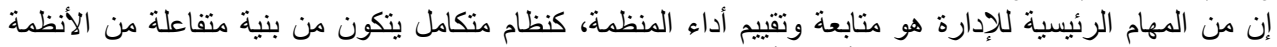

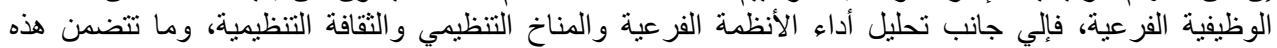

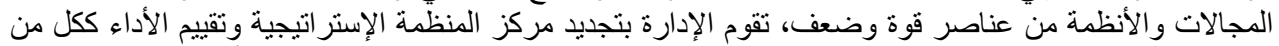

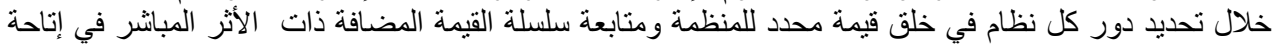

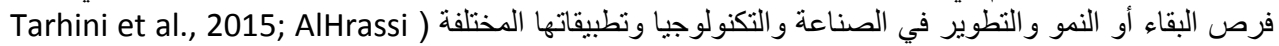
et al., 2016; Masa'deh et al., 2018a, b, c; Yassien \& Mufleh, 2017; Alenezi et al., 2017; Alkandari et al., 2017; Altamony \& Gharaibeh, 2017; Khwaldeh et al., 2017; Mikkawi \& Al-

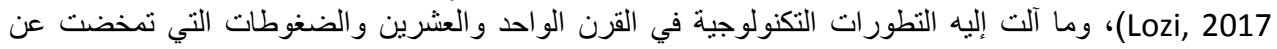

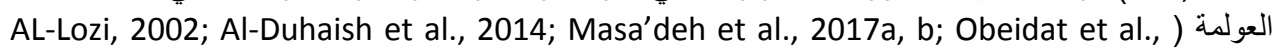
(2013, 2016, 2017; Alananzeh et al., 2018; Abualoush et al., 2018a, b

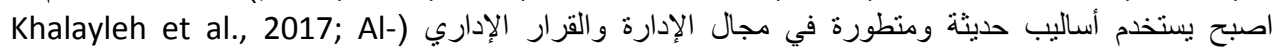
AL-Syaidh et al., 2014, 2016; ) (dalahmeh et al., 2018 ، فسارعت إلى تطوير نظم المعلومات الإدارية (Darawsheh et al., 2016; Masa'deh et al., 2015a, b والخدمات الاكترونية بمختلف القطاعات ( Masa'deh, et al., 2008; Shannak \& Obeidat, 2012; Karajeh \& Maqableh, 2014; Maqableh \& Karajeh, 2014; Al-Dmour et al., 2015; Almajali \& Maqableh, 2015; Maqableh \& Mohammed, 2015; Maqableh et al., 2015; Masa'deh, 2016; Tarhini et al., 2016, 2017a, 2017b; Almajali \& Al-Dmour, 2016; Almajali \& Tarhini, 2016; Almajali et al., 2016a, b; Aldmour et al., 2017; Obeidat et al., 2012, 2017, 2019;

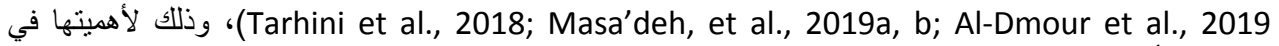

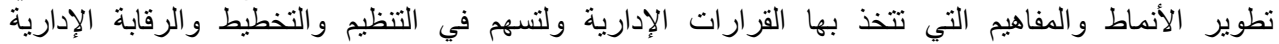

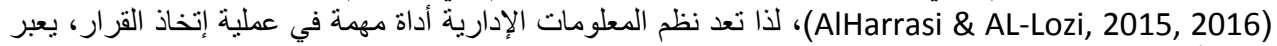

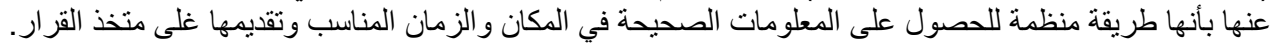


و وليه، توصي الدراسة الحاليه لبحث كيفية تفعيل القيادة الاستراتيجية واستثمار نظم المعلومات الإدارية المتطورة

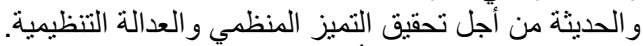

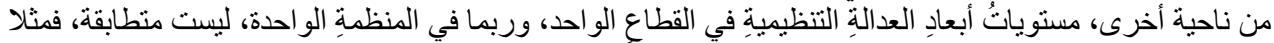

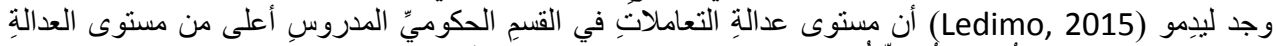

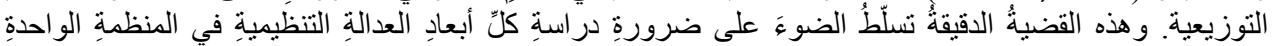

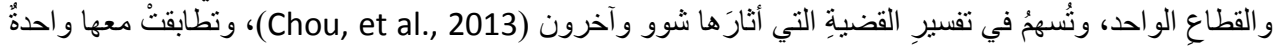

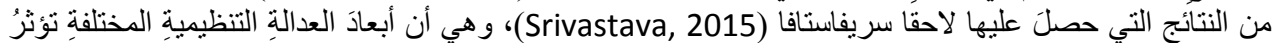

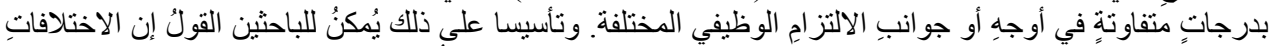

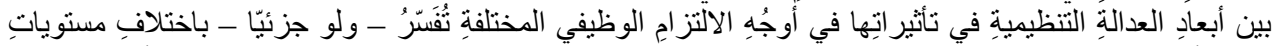

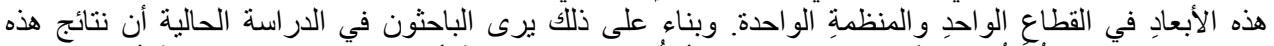

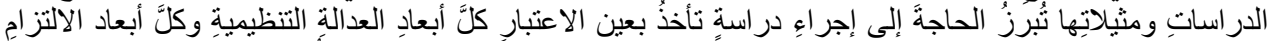

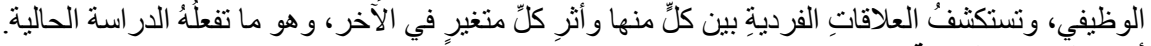

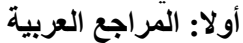

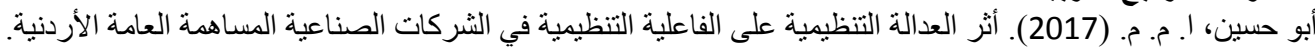

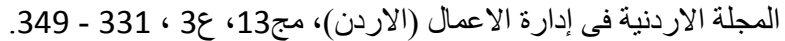

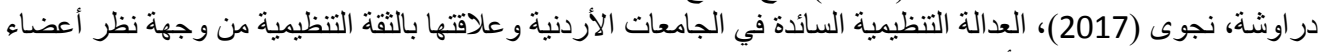
هيئة التدريس. المجلة الأردنية في العلوم التربوية، 13(3)، 373 373-388.

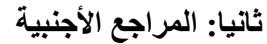

\section{Refernces}

Abualoush, S., Bataineh, K., \& Alrowwad, A. (2018a). The Role of Knowledge Management Process and Intellectual Capital as Intermediary Variables between Knowledge Management Infrastructure and Organization Performance. Interdisciplinary Journal of Information, Knowledge, and Management, 13, 279-309.

Abualoush, S., Obeidat, B., \& Tarhini, A. (2018b). The Role of Employees' Empowerment as an Intermediary Variable between Knowledge Management and Information Systems on Employees' Performance. VINE Journal of Information and Knowledge Management Systems, 48(2), 217-237.

Agarwal, R.N. (2015). Stress, Job Satisfaction and Job Commitment's Relation with Attrition with Special Reference to Indian IT Sector. Paper presented at the 9th International Management Conference "Management and Innovation for Competitive Advantage", Bucharest, Romania November 5-6, 2015.

Ajadi, O.T., \& Alade-Yussuf, F.A. (2016). Performance Indicators and Job Commitment of University Graduates during the National Youth Service Corps Programme in Oyo State, Nigeria. Asia Pacific Journal of Education, Arts and Sciences, 3(2), 1-7.

Alananzeh, O., Jawabreh, O., Al-Mahmoud, A., \& Hamada, R. (2018). The Impact of Customer Relationship Management on Tourist Satisfaction: The Case of Radisson Blue Resort in Aqaba City. Journal of Environmental Management and Tourism, 2(26), 227240. 
Al-dalahmeh, M., Khalaf, R., \& Obeidat, B. (2018). The Effect of Employee Engagement on Organizational Performance via the Mediating Role of Job Satisfaction: The Case of IT Employees in Jordanian Banking Sector. Modern Applied Science, 12(6), 17-43.

Al-Dmour, R., Al Haj Dawood, E., Al-Dmour, H., \& Masa'deh, R. (2019). The Effect of Customer Lifestyle Patterns on the Use of Mobile Banking Applications in Jordan. Int. J. Electronic Marketing and Retailing, Forthcoming.

Aldmour, R., Masa'deh, R., \& Obeidat, B. (2017). Factors Influencing the Adoption and Implementation of HRIS Applications: Are they Similar. International Journal of Business Innovation and Research, 14(2), 139-167.

Al-Dmour, R, Obeidat, B., \& Almajali, D. (2015). The Practice of HRIS Applications in Business Organizations in Jordan: An Empirical Study. 4th Scientific \& Research Conference on New Trends in Business, Management and Social Sciences (COES\&RJTK15/1).

Al-Duhaish, A., Alshurideh, M., \& Al-Zu'bi, Z. (2014). The Impact of the Basic Reference Group Usage on the Purchasing Decision of Clothes (A Field Study of Saudi Youth in Riyadh City). Dirasat: Administrative, 41(2), 205-221.

Alenezi, H., Tarhini, A., Alalwan, A., \& Al-Qirim, N. (2017). Factors Affecting the Adoption of E-Government in Kuwait: A Qualitative Study. Electronic Journal of e-Government, 15(2), 84-102.

AlHarrasi, J., \& AL-Lozi, M. (2015). The Role of Innovation Management and Technological Innovation on Organizational Effectiveness. 4th Scientific \& Research Conference on New Trends in Business, Management and Social Sciences (COES\&RJTK15/1), Istanbul, Turkey.

AlHarrasi, J., \& AL-Lozi, M. (2016). The Role of Innovation Management and Technological Innovation on Organizational Effectiveness: A Theoretical Model. Journal of Social Sciences (COES\&RJ-JSS), 5(1), 80-95.

AlHrassi, J., Al-Lozi, M., \& Irtaimeh, H. (2016). The Impact of Management Innovation and Technological Innovation on Organizational Effectiveness: An Empirical Study from Managerial Staff Perspective in Sultan Qaboos University. Journal of Social Sciences (COES\&RJ-JSS), 5(3), 309-339.

Alkandari, A., Masa'deh, R., \& Al-Lozi, M. (2017). Knowledge Management and its Role on Organizational Crisis Management: A Literature Review. Journal of Social Sciences (COES\&RJ-JSS), 6(4), 833-850.

AL-Lozi, M. (2002). Total Quality Management in the Civil Service Institutions in Jordan. Mu'tah: Humanities and Social Sciences Series, 18(4), 151-185. 
Almajali, D., \& Al-Dmour, R. (2016). The Role of Information Technology in Motivating Students to Accept E-Learning Adoption in Universities: A Case Study in Jordanian Universities. Journal of Business \& Management (COES\&RJ-JBM), 4(1), 36-46.

Almajali, D., \& Maqableh, M. (2015). Assessing the Digital Divide Status of the Jordanian Telecentre. International Journal of Communications, Network and System Sciences, 8(11), 428-439.

Almajali, D., \& Tarhini, A. (2016). Antecedents of ERP Systems Implementation Success: A Study on Jordanian Healthcare Sector. Journal of Enterprise Information Management, 29(4), 549-565.

Almajali, D., Mansour, K., \& Maqableh, M. (2016a). The Impact of Electronic Supply Chain Management Usage on Firm's Performance. International Journal of Communications, Network and System Sciences, 9(6), 280-293.

Almajali, D., Masa'deh, R., \& Al-Lozi, M. (2016b). Determinants of the Actual Use of ELearning Systems: An Empirical Study on Zarqa University in Jordan. Journal of Social Sciences (COES\&RJ-JSS), 5(2), 172-200.

AL-Syaidh, N., Al- Lozi, M., \& AlHarrasi, J. (2016). Transformational Leadership and its Role on the Effectiveness of Employees' Behavior: A Theoretical Study. Journal of Business \& Management (COES\&RJ-JBM), 4(1), 14-35.

AL-Syaidh, N., Masa'deh, R., \& Al-Zu'bi, Z. (2014). Transformational Leadership and its Impact on the Effectiveness of Employees' Behavior in the Public and Private Jordanian Hospitals. Jordan Journal of Business Administration, 11(1), 23-57.

Altamony, H., \& Gharaibeh, A. (2017). The Role of Academic Researcher to Mintzberg's Managerial Roles. International Journal of Business Management and Economic Research, 8(2), 920-925.

Aroniyiaso, O.T. (2016). Influence of Monthly Income and Position on Job Satisfaction and Commitment among Female Officers and Men in Nigeria Custom Service, Nigeria. International Journal of Research in Humanities and Social Studies, 3(11), 17-21.

Awan, M.H. (2018). The Factors Influencing Job Commitment of Teaching Staff in University of Sargodha: Mediating Role of Transformational Leadership. International Journal of Social Sciences, Humanities and Education, 2(1), 1-17.

Awolusi, O.D. (2013). Effects of Motivation on Employees' Job Commitment in the Nigerian Banking Industry: An Empirical Analysis. International Journal of Business and Innovation Research, 1(3), 1-17. 
Batool, S.S., Parveen, N., \& Batool, S.A. (2017). Emotional Intelligence and Job Commitment: Meditational Role of Job Satisfaction and Job Performance. Pakistan Business Review, 904-923.

Chou, T., Chou, S.T., Jiang, J.J., \& Klein, G. (2013). The Organizational Citizenship Behavior of IS Personnel: Does Organizational Justice Matter? Information \& Management, 50, 105-111.

Coetzee, M., Schreuder, D., \& Tladinyane, R. (2014). Employees' Work Engagement and Job Commitment: The Moderating Role of Career Anchors. Journal of Human Resource Management, 12(1), 1-12.

Darawsheh, S., ALshaar, A., \& AL-Lozi, M. (2016). The Degree of Heads of Departments at the University of Dammam to Practice Transformational Leadership style from the Point of View of the Faculty Members. Journal of Social Sciences (COES\&RJ-JSS), 5(1), 56-79.

Eskandari, M., \& Gorji, M.A. (2018). Can Work-related Stress and Job Satisfaction Affect Job Commitment among Nurses? A Cross-Sectional Study. F1000Research, 7(218), 1-10.

Güney, S., Diker, O., Güney, S., Ayranci, E., \& Solmaz, H. (2012). Effects of Organizational Communication on Work Commitment: A Case Study on a Public Agency in Ankara. Business Management Dynamics, 2(4), 18-29.

Karajeh, H., \& Maqableh, M. (2014). Security of Cloud Computing Environment. The 23rd IBIMA Conference on Vision 2020: Sustainable Growth, Economic Development, and Global Competitiveness, USA, 2202-2215.

Keller, A.C., \& Semmer, N.K. (2013). Changes in Situational and Dispositional Factors as Predictors of Job Satisfaction. Journal of Vocational Behavior, 83, 88-98.

Khalayleh, W., Masa'deh, R., \& Al-Lozi, M. (2017). Administrative Empowerment and its Role on the Work Teams Performance: A Literature Review. Journal of Social Sciences (COES\&RJ-JSS), 6(4), 851-868.

Khwaldeh, S., Al-Hadid, I., Masa'deh, R., \& Alrowwad, A. (2017). The Association between E-Services Web Portals Information Quality and ICT Competence in the Jordanian Universities. Asian Social Science, 13(3), 156-169.

Krejcie, R.V., \& Morgan, D.W. (1970). Determining Sample Size for Research Activities. Educational and Psychological Measurement, 30, 607-610.

Ledimo, O.M. (2015). An Exploratory Study of Factors Influencing Organizational Justice among Government Employees. The Journal of Applied Business Research, 31(4), 15491562. 
Maqableh, M., \& Mohammed, A. (2015). The Acceptance and Use of Computer based Assessment in Higher Education. Journal of Software Engineering and Applications, 8(10), 557.

Maqableh, M., \& Karajeh, H. (2014). Job Scheduling for Cloud Computing using Neural Networks. Communications and Network, 6(3), 191-200.

Maqableh, M., Rajab, L., Quteshat, W., Khatib, T., \& Karajeh, H. (2015). The Impact of Social Media Networks Websites Usage on Students' Academic Performance. Communications and Network, 7(4), 159-171.

Masa'deh, R. (2016). The Role of Knowledge Management Infrastructure in Enhancing Job Satisfaction at Aqaba Five Star Hotels in Jordan. Communications and Network, 8(4), 219-240.

Masa'deh, R., Alananzeh, O., Algiatheen, N., Ryati, R., Albayyari, R., \& Tarhini, A. (2017a). Employee's Perception of Implementing Green Supply Chain Management on Hotel's Economic and Operational Performance. Journal of Hospitality and Tourism Technology, 8(3), 395-416.

Masa'deh, R., Shannak, R., Maqableh, M., \& Tarhini, A. (2017b). The Impact of Knowledge Management on Job Performance in Higher Education: The Case of the University of Jordan. Journal of Enterprise Information Management, 30(2), 244-262.

Masa'deh, R., Alananzeh, O., Algudah, O., \& Tarhini, A. (2018a). The Effect of Promotional Mix on Hotel Performance during the Political Crisis in the Middle East. Journal of Hospitality and Tourism Technology, 9(1), 33-49.

Masa'deh, R., Alrowwad, A., Alkhalafat, F., Obeidat, B., \& Abualoush, S. (2018b). The Role of Corporate Social Responsibility in Enhancing Firm Performance from the Perspective of IT Employees in Jordanian Banking Sector: The Mediating Effect of Transformational Leadership. Modern Applied Science, 12(7), 1-26.

Masa'deh, R., Al-Henzab, J., Tarhini, A., \& Obeidat, B. (2018c). The Associations among Market Orientation, Technology Orientation, Entrepreneurial Orientation and Organizational Performance. Benchmarking: An International Journal, 25(8), 3117-3142.

Masa'deh, R., Hunaiti, Z., \& Bani Yaseen, A. (2008). An Integrative Model Linking ITBusiness Strategic Alignment and Firm Performance: The Mediating Role of Pursuing Innovation and Knowledge Management Strategies. Communications of the International Business Information Management Association (IBIMA) Journal.

Masa'deh, R., Gharaibeh, A., Tarhini, A., \& Obeidat, O. (2015a). Knowledge Sharing Capability: A Literature Review. 4th Scientific \& Research Conference on New Trends in Business, Management and Social Sciences (COES\&RJ-TK15/1), Istanbul, Turkey. 
Masa'deh, R., Obeidat, B., Zyod, D., \& Gharaibeh, A. (2015b). The Associations among Transformational Leadership, Transactional Leadership, Knowledge Sharing, Job Performance, and Firm Performance: A Theoretical Model. Journal of Social Sciences (COES\&RJ-JSS), 4(2), 848-866.

Masa'deh, R., Almajali, D., Alrowwad, A., \& Obeidat, B. (2019a). The Role of Knowledge Management Infrastructure in Enhancing Job Satisfaction: A Developing Country Perspective. Interdisciplinary Journal of Information, Knowledge, and Management. Forthcoming.

Masa'deh, R., Obeidat, B., Maqableh, M., \& Shah, M. (2019b). The Impact of Business Intelligence Systems on an Organization's Effectiveness: The Role of Metadata Quality from a Developing Country's View. International Journal of Hospitality \& Tourism Administration, Forthcoming.

Mikkawi, B., \& Al-Lozi, M. (2017). The Impact of Knowledge Management Infrastructure on Academic Staff Effectiveness: An Empirical Study at The University of Jordan. Jordan Journal of Business Administration, 13(1), 95-127.

Mukherjee, S., Singh, A., \& Mehrotra, S. (2016). Organizational Justice in relation to Competence, Commitment and Self Motivation. The International Journal of Indian Psychology, 3(2), 33-59.

Nakamura, S., Somemura, H., Sasaki, N., Yamamoto, M., Tanaka, M., \& Tanaka, K. (2016). Effect of Management Training in Organizational Justice: A Randomized Controlled Trial. Industrial Health, 54, 263-271.

Nicklin, J.M., McNall, L.A., Cerasoli, C.P., Strahan, S.R., \& Cavanaugh, J.A. (2014). The Role of Overall Organizational Justice Perceptions within the Four-Dimensional Framework. Social Justice Research, 27, 243-270.

Obeidat, O., Al-Suradi, M., \& Tarhini, A. (2016). The Impact of Knowledge Management on Innovation: An Empirical Study on Jordanian Consultancy Firms. Management Research Review, 39(10), 1214-1238.

Obeidat, O., El-Rimawi, S., Maqableh, M., \& Al-Jarrah, I. (2013). Evaluating the Profitability of the Islamic Banks in Jordan. European Journal of Economics, Finance and Administrative Sciences, 56, 27-36.

Obeidat, O., Hadidi, A., \& Tarhini, A. (2017). Factors Affecting Strategy Implementation: A Case Study of Pharmaceutical Companies in the Middle East. Review of International Business and Strategy, 27(3), 386-408.

Obeidat, O., Tarhini, A., \& Aqqad, N. (2019). The Relationship among Emotional Intelligence, Conflict Management Styles, and Job Performance in Jordanian Banks. International Journal of Human Resources Development and Management, Forthcoming. 
Obeidat, O., Sweis, R., Zyod, D., \& Alshurideh, M. (2012). The Effect of Perceived Service Quality on Customer Loyalty in Internet Service Providers in Jordan. Journal of Management Research, 4(4), 224-242.

Olaojo, P.O., \& Oyeboade, J.A. (2016). Social Support, Work Motivation and Work Commitment of Library Personnel in Selected Private University Libraries in South-West, Nigeria. Qualitative and Quantitative Methods in Libraries, 5, 11-22.

Oludeyi, O.S. (2015). A Reviewof Literature on Work Environmentand Work Commitment: Implication for Future Research in Citadels of Learning. Journal of Human Resource Management, 18(2), 32-46.

Oludeyi, O.S., Momoh, A.M., \& Akinsanya, A.O. (2018). Campus Environmental Factors and Job Commitment of Staff in State Universities in Ogun Southwest Nigeria. HSS, VII(1), 42-61.

Rai, G.S. (2013). Impact of Organizational Justice on Satisfaction, Commitment and Turnover Intention: Can Fair Treatment by Organizations make a Difference in their Workers' Attitudes and Behaviors? International Journal of Human Sciences, 10(2), 260284.

Rajabi, A., \& Najafzadeh, M.R. (2015). The Relationship between Organizational Justice and Organizational Commitment of Physical Education Teachers of Tabriz. International Journal of Basic Sciences \& Applied Research, 4(5), 255-259.

Rezaeizadeh, M., Monfared, J.H., \& Ghasemipour, M.R. (2015). Surveying the Effect of Organizational Justice on Employee Commitment in Iranian Petrochemical Company (Case Study: Imam Khomeini Petrochemical). Applied Mathematics in Engineering, Management and Technology, 3(5), 16-24.

Saeed, I., Junaid, M., Fatima, T., Abrar, A., Gohar, M., \& Benazir, S. (2017). Effect of Organizational Justice on Job Outcomes: Mediating Role of Affective Commitment. Journal of Management Research, 3(2), 192-208.

Salajeghea, S., \& Asgharpour, L. (2014). Studying the Relationship between Organizational Justice and Staff Job Commitment a Case Study of Kerman State Tax Administration Staff. Scientific Journal of Review, 3(8), 898-908.

Shannak, R., \& Obeidat, B. (2012). Culture and the Implementation Process of Strategic Decisions in Jordan. Journal of Management Research, 4 (4), pp. 257-281.

Srivastava, U.R. (2015). Multiple Dimensions of Organizational Justice and Work-Related Outcomes among Health-Care Professionals. American Journal of Industrial and Business Management, 5, 666-685. 
Swarnalatha, S.S. (2016). Work Commitment of Secondary School Teachers. The International Journal of Indian Psychology, 3(4), 84-89.

Tarhini, A., Alalwan, A., Al-Qirim, N., \& Algharabat, R. (2018). An Analysis of the Factors Influencing the Adoption of Online Shopping. International Journal of Technology Diffusion (IJTD), 9(3), 68-87.

Tarhini, A., Al-Badi, A., Almajali, M., \& Alrabayaah, S. (2017a). Factors Influencing Employees' Intention to Use Cloud Computing. Journal of Management and Strategy, 8(2), 47.

Tarhini, A., Al-Busaidi, K., Bany Mohammed, A., \& Maqableh, M. (2017b). Factors Influencing Students' Adoption of E-Learning: A Structural Equation Modeling Approach. Journal of International Education in Business, 10(2), 164-182.

Tarhini, A., Bany Mohammed, A., \& Maqableh, M. (2016). Modeling Factors Affecting Student's Usage Behaviour of E-Learning Systems in Lebanon. International Journal of Business and Management, 11(2), 299.

Tarhini, A., Mgbemena, C., \& Trab, MSA. (2015). User Adoption of Online Banking in Nigeria: A Qualitative Study. Journal of Internet Banking and Commerce, 20(3), 1-8.

Umoh, G.I., Amah, E., \& Wokocha, I.H. (2014). Employee Benefits and Continuance Commitment in the Nigerian Manufacturing Industry. IOSR Journal of Business and Management, 16(2), 69-74.

Wołowska, A. (2014). Determinants of Organizational Commitment. Human Resources Management \& Ergonomics, VII(1), 129-146.

Yassien, E., \& Mufleh, M. (2017). The Impact of ERP System's Usability on Enterprise Resource Planning Project Implementation Success via the Mediating Role of User Satisfaction. Journal of Management Research, 9(3), 49-71. 